\title{
Implementation of Nonlocal Pansharpening Image Fusion
}

\author{
Joan Duran ${ }^{1}$, Antoni Buades ${ }^{2}$, Bartomeu Coll ${ }^{3}$, Catalina Sbert ${ }^{4}$ \\ ${ }^{1}$ Universitat de les Illes Balears, Spain (joan.duran@uib.es) \\ ${ }^{2}$ Universitat de les Illes Balears, Spain (toni.buades@uib.es) \\ ${ }^{3}$ Universitat de les Illes Balears, Spain (tomeu.coll@uib.es) \\ ${ }^{4}$ Universitat de les Illes Balears, Spain (catalina.sbert@uib.es)
}

Communicated by Vicent Caselles and Gabriele Facciolo

Demo edited by Jose-Luis Lisani

\begin{abstract}
This paper focuses on the implementation of the pansharpened image fusion technique proposed in the companion paper [A Nonlocal Variational Model for Pansharpening Image Fusion, SIAM Journal on Imaging Sciences, 2014, to appear]. Pansharpening refers to the process of inferring a high resolution multispectral image from a high resolution panchromatic image and low resolution multispectral one. Although quite successful in terms of relative error, state-of-the-art pansharpening methods still introduce relevant color artifacts. The variational pansharpening model proposed by Duran et al. incorporates a nonlocal regularization term that takes advantage of image self-similarity, leading to significant reduction of the above-mentioned color artifacts.
\end{abstract}

\section{Source Code}

ANSI $\mathrm{C}$ source code to produce the same results as the demo is accessible at the IPOL web page of this article $^{1}$.

Keywords: image fusion; pansharpening; multispectral imagery; neighborhood filter; nonlocal operators; energy functional

\footnotetext{
${ }^{1}$ https://doi.org/10.5201/ipol.2014.98
} 


\section{Introduction}

Many Earth observation satellites, such as SPOT, Landsat, IKONOS or QuickBird, provide continuously growing quantities of remote sensing images useful for a wide range of tasks. For example, satellite images are used in feature detection, land cover classification, water depth estimation, soil moisture content, vegetation mapping or many military tasks such as mission planning, navigation and targeting.

Digital color images are usually represented by three color values at each pixel. However, most common cameras use a CCD sensor device measuring a single color per pixel (either red, green or blue). The other two color values of each pixel must be interpolated from the neighboring pixels in the so-called demosaicking process. The selected configuration of the CCD sensor usually follows the CFA Bayer where, out of a group of four pixels, two are green, one is red and one is blue. Most satellites use a different acquisition system that decouples the acquisition of a panchromatic image at high spatial resolution from the acquisition of a multispectral image at lower spatial resolution. The design of multispectral sensors with better resolution is limited by technical constraints of on-board storage and by bandwidth transmission of the images from the satellite to the Earth.

High spatial resolution is necessary to correctly detect shapes, edges and structures. On the contrary, different types of land are better classified using images with multiple spectral bands. Accordingly, the goal of pansharpening image fusion is to integrate the geometric detail of the panchromatic image and the precise spectral information of the multispectral image to produce a high resolution multispectral image. Figure 1 illustrates the pansharpening problem.

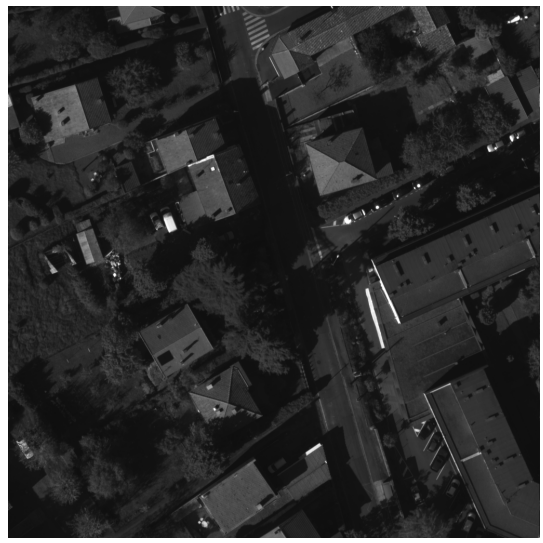

Panchromatic image

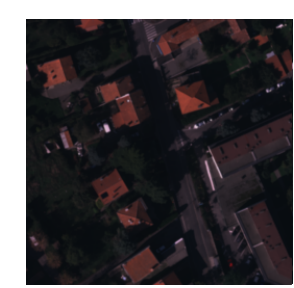

Multispectral image

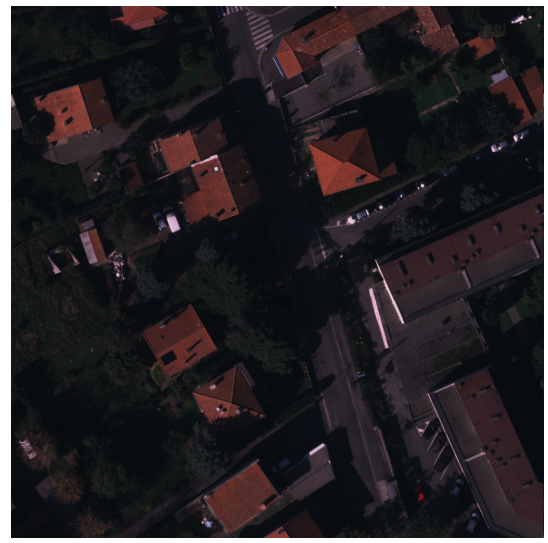

Pansharpened image

Figure 1: Description of the pansharpening problem. Most satellites provide a high-resolution grayscale image that contains information about shapes, features and structures, and a multispectral image at lower spatial resolution. The goal of pansharpening is to infer a high resolution multispectral image from the previous data.

The pansharpening has been an intensive field of research during the last decades. Initial methods imposed the final color image to match the luminance of the panchromatic image while maintaining the low frequencies of the initial chromatic components. This is the case of the componentsubstitution techniques, for instance the Intensity-Hue-Saturation (IHS) $[12,5,17,21]$ and the Principal Component Analysis transforms (PCA) [6, 9, 19], or of relative spectral contribution methods, such as intensity modulation [7] and Brovey's method [23]. Several models using discrete wavelet transforms [26, 10, 27, 16, 25] or Laplacian pyramid algorithms [24, 1] have also been proposed. Some recently proposed variational models determine the pansharpened image through the minimization of specific energy functionals [2, 15]. Pansharpening techniques have been extensively reviewed in the literature $[22,20,8]$. 
Duran et al. [3] proposed a variational pansharpening formulation inspired on the NL-means denoising algorithm [4]. The functional consists of two fidelity terms, which force the pansharpened image to be consistent with panchromatic and multispectral data, and a nonlocal regularization term which acts as a neighborhood filter. The panchromatic image is used to derive nonlocal relationships among patches describing the geometry of the desired pansharpened image. This geometry being available through the panchromatic image, the problem reduces to a linear quadratic system as proposed in [11].

In this paper, we first introduce the nonlocal functional form of the NL-means after which we present the pansharpening model proposed by Duran et al. [3]. Then we focus on the description and implementation of the derived algorithm. Lastly, a detailed performance comparison with some of state-of-the-art methods for pansharpening is presented.

\section{Nonlocal Regularization}

The NL-means algorithm [4] denoises a pixel $x \in \Omega$ by averaging the values of all pixels whose Gaussian neighborhood looks like the neighborhood of $x$ :

$$
\mathrm{NL}[u](x)=\frac{1}{C(x)} \int_{\Omega} e^{-\frac{d_{\rho}(u(x), u(y))}{h^{2}}} u(y) d y, \quad \forall x \in \Omega,
$$

where

$$
C(x)=\int_{\Omega} e^{-\frac{d_{\rho}(u(x), u(y))}{h^{2}}} d y
$$

is a normalizing factor and the distance $d_{\rho}$ is given by

$$
d_{\rho}(u(x), u(y))=\int_{\Omega} G_{\rho}(t)|u(x+t)-u(y+t)|^{2} d t .
$$

In this framework, $G_{\rho}$ is a Gaussian kernel and $h$ acts as a filtering parameter. The parameter $h$ controls the decay of the exponential function and, therefore, the decay of the weights as a function of the Euclidean distances.

Kindermann et al. [13] were the first to formulate the NL-means algorithm as a variational method. They interpreted the NL-means, and the neighborhood filters in general, as regularizations based on nonlocal functionals in the general form

$$
\int_{\Omega} \int_{\Omega} g\left(\frac{|u(x)-u(y)|^{2}}{h^{2}}\right) w(|x-y|) d x d y
$$

where $w(|x-y|)$ is a spatial weight function. Similar approaches have been introduced for many image processing applications where classical TV minimization [18] is replaced by nonlocal regularization terms. Gilboa and Osher [11] considered the general quadratic nonlocal functional

$$
J(u)=\int_{\Omega} \int_{\Omega}(u(x)-u(y))^{2} w_{u_{0}}(x, y) d x d y,
$$

where $w_{u_{0}}(x, y)$ now depends on the initial image $u_{0}$. The weight function $w_{u_{0}}$ is always assumed nonnegative and symmetric. Gilboa and Osher [11] minimized $J(u)$ with a gradient descent method derived from the associated Euler-Lagrange equation, that is,

$$
u_{n+1}(x)=\frac{1}{C(x)} \int_{\Omega} u_{n}(y) w_{u_{0}}(x, y) d y,
$$

where $C(x)=\int_{\Omega} w_{u_{0}}(x, y) d y$ is a normalizing factor and $u_{0}$ is given as an initial condition of the process. This formulation is a linear and powerful tool for image processing since the weight distribution is independent of $u$ and there is no restriction on the way the initial condition is computed. 


\section{The Pansharpening Model}

The pansharpening model proposed by Duran et al. [3] is based on a variational formulation and it is related to the works of Ballester et al. [2] and of Gilboa and Osher [11].

Let us denote by $\vec{u}=\left(u_{1}, \ldots, u_{M}\right)$ the true multispectral image defined on an open bounded domain $\Omega \subset \mathbb{R}^{N}$, where $M$ is the number of spectral channels. Each coordinate $u_{m}: \Omega \rightarrow \mathbb{R}$, $1 \leq m \leq M$, represents the intensity corresponding to the $m$-th spectral band. For color images, $M=3$ and the usual red, green and blue channels are considered. The available data is composed by the panchromatic image $P$ defined in the same domain $\Omega$ and the observed spectral components, $\vec{u}^{S}=\left(u_{1}^{S}, \ldots, u_{M}^{S}\right)$, defined on a sampled grid $S \subseteq \Omega, u_{m}^{S}: S \rightarrow \mathbb{R}$, whose points will be called the low-resolution pixels.

We propose to minimize a functional consisting of a nonlocal regularization term and two fidelity terms, which force the pansharpened image to be consistent with panchromatic and low-resolution multispectral data.

Regularization term. The panchromatic image is used to derive nonlocal relationships among patches. Accordingly, we introduce a quadratic regularization term as in (1) with the weight distribution given by

$$
\omega(x, y)=\frac{1}{C(x)} e^{-\frac{d_{\rho}(P(x), P(y))}{h^{2}}}
$$

where

$$
C(x)=\int_{\Omega} e^{-\frac{d_{\rho}(P(x), P(y))}{h^{2}}} d y .
$$

Note that $\omega(x, y)$ satisfies the usual conditions $0 \leq \omega(x, y) \leq 1$ and $\int_{\Omega} \omega(x, y) d y=1$, but the normalization using (3) breaks down the symmetry of the weights between two given pixels in the image. By using (2)-(3), the geometry of the panchromatic is transported to the pansharpened image.

Panchromatic constraint. We assume that the panchromatic image and the spectral bands are geometrically aligned. Thus $P$ should be a linear combination of the different channels of the multispectral image, that is,

$$
P(x)=\sum_{m=1}^{M} \alpha_{m} u_{m}(x), \quad \forall x \in \Omega,
$$

where $\alpha_{m} \geq 0,1 \leq m \leq M$, and $\sum_{m} \alpha_{m}=1$.

Spectral constraint. The low-resolution image is assumed to be related to the high-resolution one by a low-pass filtering followed by subsampling, which writes as

$$
k_{m} * u_{m}(p)=u_{m}^{S}(p), \quad \forall p \in S, \quad \forall 1 \leq m \leq M,
$$

where $k_{m}$ is the impulse response for the spectral channel $u_{m}$. In order to preserve the frequency information from the original low-resolution multispectral image $\vec{u}^{S}$, the following term is considered in the energy functional

$$
\int_{\Omega} \Pi_{S} \cdot\left(k_{m} * u_{m}(x)-u_{m}^{\Omega}(x)\right)^{2} d x, \quad \forall 1 \leq m \leq M
$$

where $\Pi_{S}=\sum_{p \in S} \delta_{p}$ is a Dirac's comb defined by the sampling grid $S$, and each $u_{m}^{\Omega}$ denotes an arbitrary extension of $u_{m}^{S}$ as a continuous function from $S$ to $\Omega$. This integral of a sum of Dirac's is unambiguous as we assume that no point of $S$ belongs to the boundary of $\Omega$. Furthermore, since the integrand term is multiplied by $\Pi_{S}$, the integral expression (4) does not depend on the particular extension $u_{m}^{\Omega}$. 
By taking into account the regularization term and the constraints imposed by the data generation model, Duran et al. [3] propose to minimize the following energy functional

$$
\begin{aligned}
J(\vec{u}) & =\frac{1}{2} \sum_{m=1}^{M} \int_{\Omega} \int_{\Omega}\left(u_{m}(x)-u_{m}(y)\right)^{2} \omega(x, y) d x d y \\
& +\frac{\lambda}{2} \int_{\Omega}\left(\sum_{m=1}^{M} \alpha_{m} u_{m}(x)-P(x)\right)^{2} d x \\
& +\frac{\mu}{2} \sum_{m=1}^{M} \int_{\Omega} \Pi_{S} \cdot\left(k_{m} * u_{m}(x)-u_{m}^{\Omega}(x)\right)^{2} d x
\end{aligned}
$$

where $\lambda, \mu>0$ are weighting parameters. The same authors [3] show that there exists an unique minimizer for (5) in a specific class of functions linked to a weighted $L^{2}$ space. The associated Euler-Lagrange equation to be solved for each channel $u_{m}, 1 \leq m \leq M$, is given by

$$
-L u_{m}(x)+\lambda \alpha_{m}\left(\sum_{k=1}^{M} \alpha_{k} u_{k}(x)-P(x)\right)+\mu\left(k_{m}^{\top} *\left(\Pi_{S} \cdot\left(k_{m} * u_{m}-u_{m}^{\Omega}\right)\right)\right)(x)=0, \quad \forall x \in \Omega,
$$

where $k_{m}^{\top}$ is the kernel adjoint defined as $k_{m}^{\top}(x)=k_{m}(-x)$ and the integral operator $L$ is given by

$$
L u_{m}(x)=\int_{\Omega}\left(u_{m}(y)-u_{m}(x)\right)(\omega(x, y)+\omega(y, x)) d y, \quad \forall 1 \leq m \leq M
$$

\section{Discrete Algorithm}

Let the panchromatic image $P$ be defined on a discrete grid $I=\{0,1, \ldots, N-1\} \times\{0,1, \ldots, N-1\}$ and $p=\left(p_{i}, p_{j}\right) \in I$ denote an image pixel. Let the spectral components $u_{1}^{S}, \ldots, u_{M}^{S}$ be defined on a lower resolution grid $S$ of size $\frac{N}{s} \times \frac{N}{s}$, where $s$ is the sampling factor.

Applying the gradient descent method to minimize (5) computes the solution of (6) iterating the equation

$$
\begin{aligned}
u_{m}^{(n+1)}(p) & =u_{m}^{(n)}(p)-\Delta t \sum_{q \in I}\left(u_{m}^{(n)}(p)-u_{m}^{(n)}(q)\right)(\omega(p, q)+\omega(q, p)) \\
& -\Delta t \lambda \alpha_{m}\left(\sum_{k=1}^{M} \alpha_{k} u_{m}^{(n)}(p)-P(p)\right)-\Delta t \mu\left(k_{m}^{t} *\left(\Pi_{S} \cdot\left(k_{m} * u_{m}^{(n)}-u_{m}^{\Omega}\right)\right)\right)(p), \quad \forall p \in I,
\end{aligned}
$$

for each band $u_{m}, 1 \leq m \leq M$. In this setting, $n \geq 0$ is the iteration number, $\Delta t>0$ stands for the artificial time step in the descent direction and $u_{m}^{\bar{\Omega}}$ is an arbitrary extension of the low-resolution band $u_{m}^{S}$ to the whole grid $I$ by means of, for instance, a simple replication by $s$ factor. Recall that $\Pi_{S}$ indicates which of the pixels in the high resolution image we actually know from the low resolution image. Accordingly, the discrete version of $\Pi_{S}$ is considered here as a $N \times N$ matrix such that $\Pi_{S}\left(p_{i}, p_{j}\right)=1$ if $\left(p_{i}, p_{j}\right) \in S$ and $\Pi_{S}\left(p_{i}, p_{j}\right)=0$ otherwise. Note that this formulation requires an initialization of each $u_{m}$.

For computational purposes, the regularity term is restricted to pixels at a certain distance from the current pixel (support zone). That is, the weight distribution $\omega(p, q)$ will be zero for pixels such that $\|p-q\|_{\infty}>K$, for a certain positive parameter $K$. Therefore, the similarity between pixels $p$ and $q$ is measured as

$$
\omega(p, q)= \begin{cases}\frac{1}{C(p)} e^{-\frac{1}{h^{2}} \sum_{t \in \mathcal{N}_{0}}\|P(p+t)-P(q+t)\|^{2}} & \text { if }\|p-q\|_{\infty} \leq K \\ 0 & \text { otherwise }\end{cases}
$$


with the normalizing factor $C(p)$ defined by

$$
C(p)=\sum_{\left\{q:\|q-p\|_{\infty} \leq K\right\}} e^{-\frac{1}{h^{2}} \sum_{t \in \mathcal{N}_{0}}\|P(p+t)-P(q+t)\|^{2}},
$$

where $\mathcal{N}_{0}$ is an $l \times l$ discrete window centered at $(0,0)$ (comparison window). Note that the Gaussian kernel $G_{\rho}$ introduced in the mathematical formulation is not considered here as it is only necessary when the size of the window increases considerably. Furthermore, the weight of the reference pixel, $\omega(p, p)$, is set to the maximum of the weights $\omega(p, q)$ for $q \neq p$. This setting avoids the excessive weighting of the reference point. Due to the fast decay of the exponential kernel, large Euclidean distances yield nearly zero weights, acting as an automatic threshold. The decay of the exponential functions, and thus the decay of the weights, is controlled by the parameter $h$.

In (7) note that, since the weights are computed on the panchromatic image, they are not modified during the evolution procedure, so they are computed only once. The complexity associated to the precomputation of the weights puts this algorithm at a computational cost higher than basic pansharpening methods. Multiresolution and preselection strategies can be used to further accelerate the patch comparisons $[4,14]$.

The whole algorithm is described by the pseudo-code in algorithm 1, which takes as input images the high-resolution panchromatic, the extension of the multispectral data to the whole grid and an arbitrary initialization. The first step of the proposed algorithm consists in computing the weight distribution on the panchromatic image. After that, the solution of the pansharpening problem is obtained by iterating equation (7) for a certain number of iterations.

\section{$5 \quad$ Experimental Results}

In this section we present experimental results illustrating the behavior of algorithm 1 for pansharpening image fusion. Experiments were performed on natural images and on full color aerial images furnished to us by CNES, Centre National d'Études Spatiales. In both cases we simulated panchromatic and subsampled spectral components from the "ground truth" reference images in the following terms. The panchromatic image was obtained by the average of the available channels, that is, the mixing coefficients were set to $\alpha_{m}=\frac{1}{M}, 1 \leq m \leq M$. The low-resolution multispectral image was simulated by Gaussian convolution followed by subsampling. We used a sampling factor $s=4$ with a corresponding Gaussian convolution of standard deviation $\sigma=2.2$.

First, we present a detailed comparison of the proposed algorithm with some relevant state-ofthe-art techniques applied to natural images. We shall compare with IHS [12], Brovey [23], Waveletsbased [26] and P+XS [2]. The initialization used for the IHS method is a simple interpolation by splines, whereas all other algorithms are initialized with the pansharpened image provided by IHS. The parameters of our algorithm were selected as follows: $\lambda=100, \mu=100 \cdot s^{2}$ (the parameter is normalized depending on the sampling factor $s$ ), $K=3$ (the size of the support zones is $7 \times 7$ pixels), $l=3$ (the size of the comparison window is $3 \times 3$ pixels) and $\Delta t=0.01$. The filtering parameter controlling the decay of the weights was selected depending on the sampling factor, namely $h=6$ if $s=4$ and $h=1.25$ if $s=2$. As stopping criterion, we used a tolerance value of $\varepsilon=10^{-3}$ for the relative error between two consecutive iterations. Anyway, we stopped the algorithm after $N_{i t e r}=100$ iterations even if the tolerance was not reached. The extended image $\vec{u}^{\Omega}$ required in the scheme (7) was constructed by means of a simple replication by a factor $s$.

Figures 2 and 3 illustrate how the colors from the original low resolution multispectral images are not necessarily preserved. The pansharpened image provided by the wavelet-based technique presents several color artifacts which mainly appear at edges. IHS and Brovey give results with high spatial resolution, however, a loss of spectral information occurs. For instance, the color of the balloon basket 


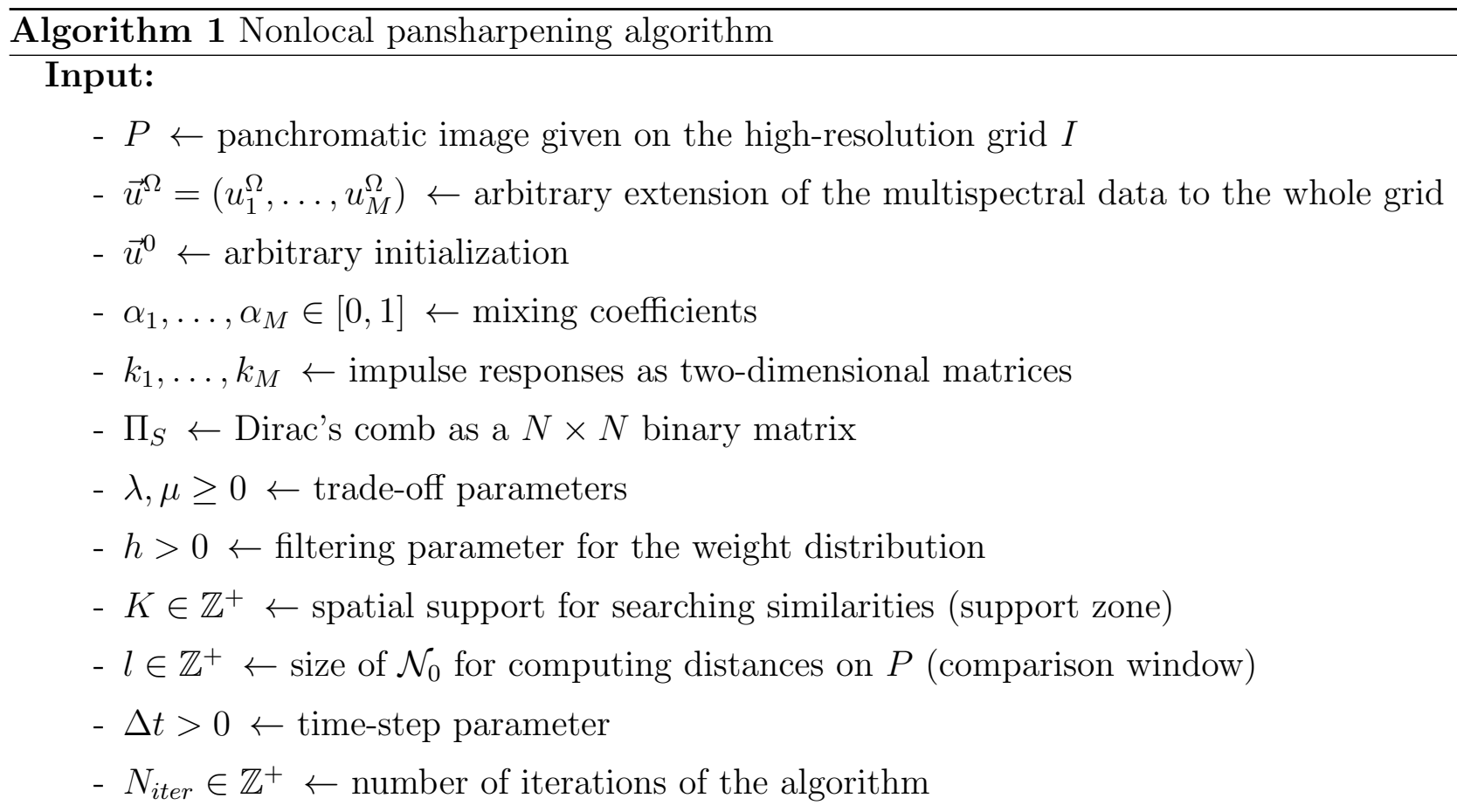

Output: pansharpened image $\vec{u}=\left(u_{1}, \ldots u_{M}\right)$

for all pixel $p \in I$ do $\quad \triangleright$ Pre-computation of the weights on the panchromatic image $C(p) \leftarrow 0$

for all pixel $q \in I, q \neq p$ do

if $\|p-q\| \leq K$ then

$$
\begin{aligned}
& \omega(p, q) \leftarrow e^{-\frac{1}{h^{2}} \sum_{t \in \mathcal{N}_{0}}\|P(p+t)-P(q+t)\|^{2}} \\
& C(p) \leftarrow C(p)+\omega(p, q)
\end{aligned}
$$

else

\section{end if}

$$
\omega(p, q) \leftarrow 0
$$

\section{end for}

$\omega(p, p) \leftarrow \max \{w(p, q): q \in I, q \neq p\}$

for all pixel $q \in I$ such that $\|p-q\| \leq K$ do

\section{end for}

$$
\omega(p, q) \leftarrow \frac{\omega(p, q)}{C(p)}
$$

\section{end for}

$$
\begin{aligned}
& \vec{u} \leftarrow \vec{u}^{0} \\
& n \leftarrow 1
\end{aligned}
$$

while $n \leq N_{\text {iter }}$ do

for all spectral channel $1 \leq m \leq M$ do

for all pixel $p \in I$ do

$$
\begin{aligned}
u_{m}(p) \leftarrow u_{m}(p) & -\Delta t \sum_{q \in I}\left(u_{m}(p)-u_{m}(q)\right)(\omega(p, q)+\omega(q, p)) \\
& -\Delta t \lambda \alpha_{m}\left(\sum_{k=1}^{M} \alpha_{k} u_{m}(p)-P(p)\right) \\
& -\Delta t \mu\left(k_{m}^{\top} *\left(\Pi_{S} \cdot\left(k_{m} * u_{m}-u_{m}^{\Omega}\right)\right)\right)(p)
\end{aligned}
$$

end for

\section{end for}

$n \leftarrow n+1$

\section{end while}

return $\vec{u}=\left(u_{1}, \ldots u_{M}\right)$. 
in figure 2 has clearly changed from brownish to grayish. A similar spectral distortion is also apparent in the result provided by $\mathrm{P}+\mathrm{XS}$. On the other hand, the proposed algorithm meaningfully reduces color artifacts outperforming all the compared techniques. This visual comparison is supported by the Root-Mean-Squared Error (RMSE) computed in RGB coordinates between the reference image and each of the results obtained after applying the above-mentioned pansharpening techniques. If we denote by $\vec{u}^{R}=\left(u_{1}^{R}, u_{2}^{R}, u_{3}^{R}\right)$ the reference image, then the formula for RMSE is given by

$$
\mathrm{RMSE}=\frac{1}{3} \sum_{m=1}^{3} \sqrt{\frac{\sum_{p \in I}\left(u_{m}(p)-u_{m}^{R}(p)\right)^{2}}{N^{2}}} .
$$

Brovey, $\mathrm{P}+\mathrm{XS}$ and IHS have a similar performance in terms of RMSE, their error being larger than the proposed one.

Figure 4 illustrates the preservation of the spatial information contained in the panchromatic image. For that purpose, the grayscale versions of the fused images were computed. All results displayed in figure 4 compare favorably to the original panchromatic image, so that edges, textures and other geometric details are highly preserved. We want to emphasize that algorithm 1 also performs a slight denoising since it reduces the inherent noise that appears, for instance, at the bow hull of the boat.

We test now all pansharpening techniques under comparison on a multispectral aerial image using three bands (red, green and blue). The goal is to check if the self-similarity underlying the model proposed by Duran et al. [3] holds for aerial images as natural images do. Figure 5 illustrates a comparative visual quality assessment of fused images. For a better visual analysis, we display the difference images between the reference one and the pansharpened results. Once again, IHS, Brovey, Wavelet-based and $\mathrm{P}+\mathrm{XS}$ techniques suffer from spectral distortion. Indeed, color saturation is significantly reduced on the results provided by these methods, see for instance the red cars or the thin wall appearing in the images. The algorithm discussed in this paper outperforms all other techniques since the difference image displayed in figure 5 contains less amount of information. Note that the proposed algorithm is also the best one in terms of root-mean-squared error.

Finally, we tested algorithm 1 on an aerial hyperspectral image furnished by the CNES. The low-resolution hyperspectral image has four channels (red, green, blue and near-infrared) with a resolution corresponding to one quarter of the panchromatic one. Furthermore, the algorithm was now initialized by a simple replication of each spectral component. Figure 6 shows the reference, the low-resolution and the pansharpened color images involving red, green and blue channels. It also displays false color images using the near-infrared, red and green spectral bands (in this order) in place of the usual RGB. At a glance, the results give good spatial resolution since the images look clear. Furthermore, the spectral information is highly preserved and no artifacts are perceptible.

\section{On Line Demo}

An online demo of the nonlocal variational model is available at the web page of this article ${ }^{2}$. The purpose is to simulate the pansharpening problem and to solve it by means of the proposed algorithm. The ANSI C source code (documented) used in this on line demo is also available at the web page.

The program inputs are the RGB image and the sampling factor $(s=2$ or $s=4)$. The other parameters are fixed as indicated previously, but the users can download the source code and try by themselves. Furthermore, one can upload its own input image or use any of the images available on the demo page. The proposed images have almost no noise. They were obtained by taking good quality photographs in full daylight and then zooming them out by an 8-factor.

\footnotetext{
${ }^{2}$ https://doi.org/10.5201/ipol.2014.98
} 
The implementation simulates the panchromatic and low-resolution multispectral images from the input. The initialization is then computed by IHS transform and the extended image is given by a simple replication of the low-resolution image by a factor $s$. The algorithm finally computes the high resolution multispectral image.

The outputs are the reference RGB image, the panchromatic image, the low-resolution image, the initialization provided by IHS, the pansharpened image, the residual images corresponding to the initialization and the solution (difference between the corresponding image and the reference one with stretched dynamic on $[0,255]$ by an affine contrast change), and the RMSE of both the initialization and the pansharpened images.

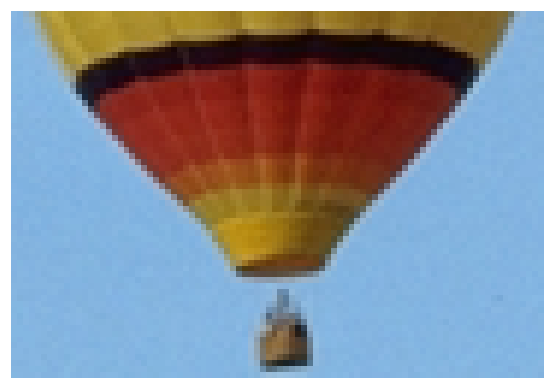

Original

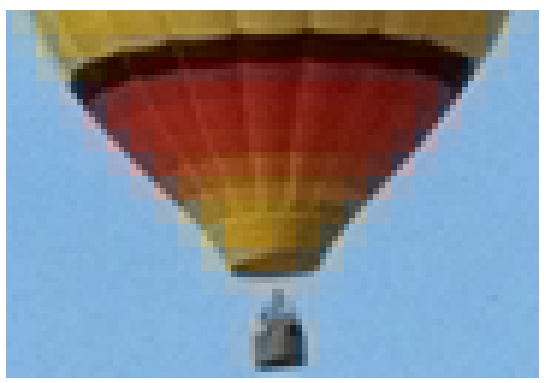

Wavelets

$\mathrm{RMSE}=4.04$

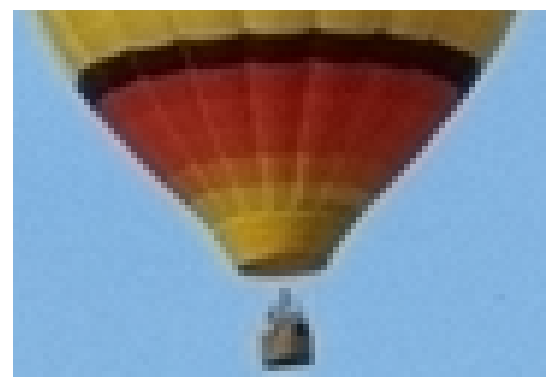

IHS

$\mathrm{RMSE}=2.75$

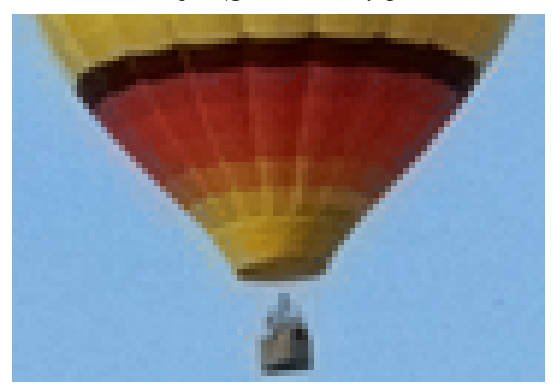

$\mathrm{P}+\mathrm{XS}$

$\mathrm{RMSE}=2.73$

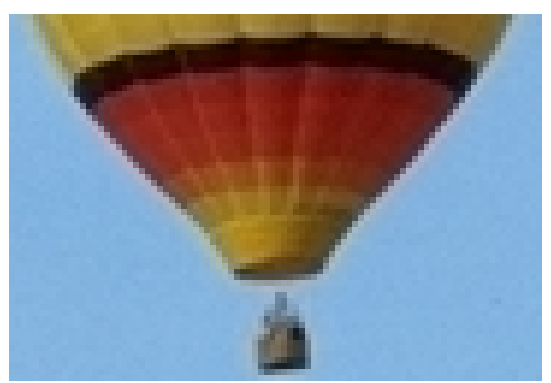

Brovey

$\mathrm{RMSE}=2.76$

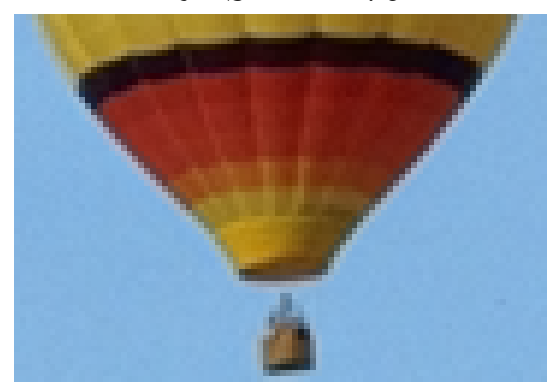

Duran et al.

$\mathrm{RMSE}=1.31$

Figure 2: Original color image and the pansharpened images provided by each technique, with sampling factor $s=4$. We also exhibit the RMSE values between the reference image and each pansharpened result. All techniques perform similarly in terms of spatial quality or geometry except for the wavelet-based one which presents artifacts at the edges of the balloon. In terms of spectral quality, the model proposed by Duran et al. [3] outperforms all other methods. Indeed, the color of the balloon basket has changed from brownish to grayish in the results provided by IHS, Brovey, Wavelet-based and P+XS techniques. Similar effects can also be observed on the balloon stripes where red and yellow are mixed resulting on a browner stripe. All these color artifacts are reduced with the proposed algorithm which is able to better preserve the original colors.

\section{Acknowledgements}

The authors were supported by the Ministerio de Ciencia e Innovación under grant TIN2011-27539. During this work, J.D. benefited from a fellowship of the Conselleria d'Educació, Cultura i Universitats of the Govern de les Illes Balears for the realization of his Ph.D. thesis, which has been selected under an operational program co-financed by the European Social Fund. 

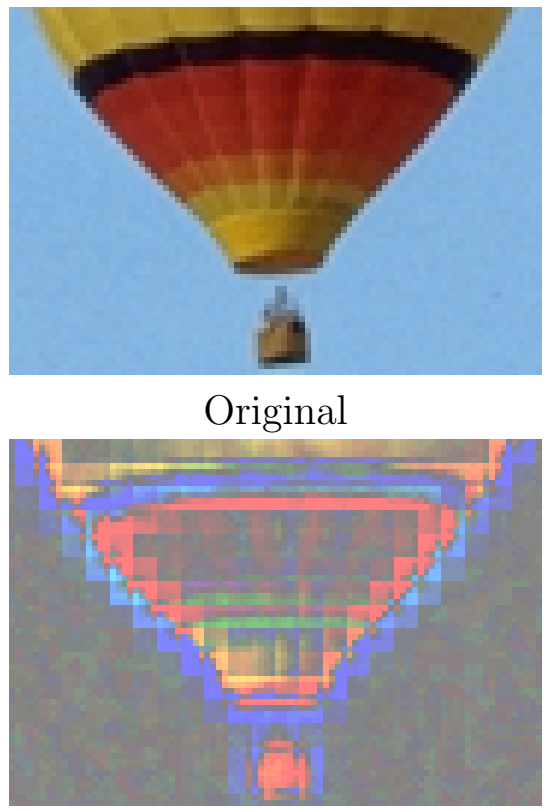

Wavelets

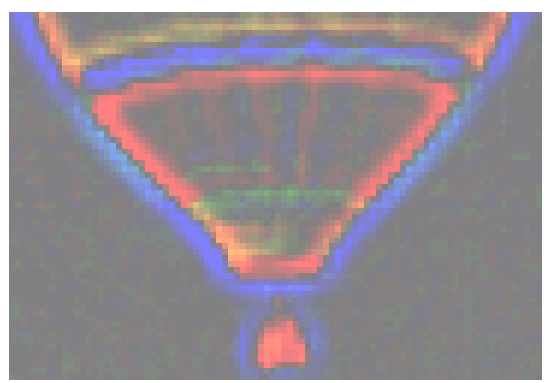

IHS

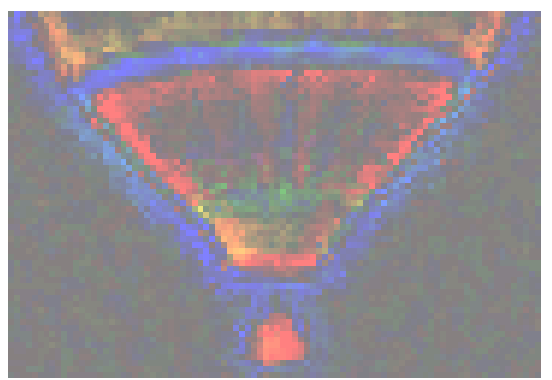

$\mathrm{P}+\mathrm{XS}$

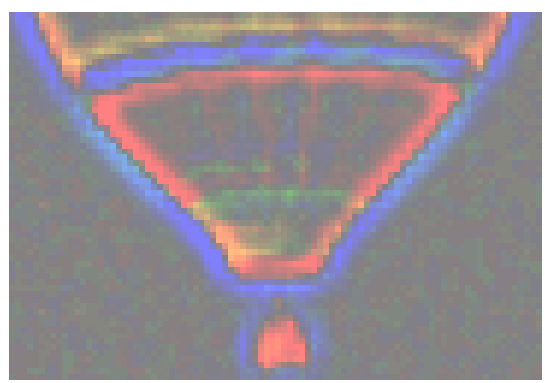

Brovey

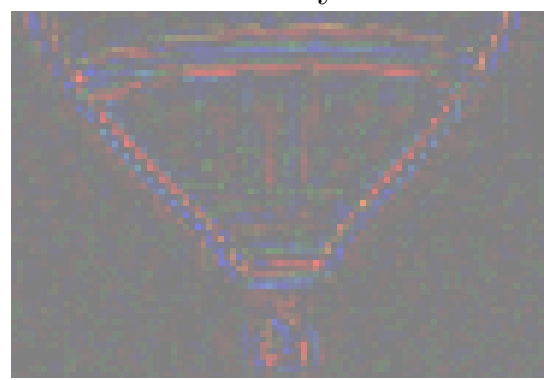

Duran et al.

Figure 3: Difference images between the original and the pansharpened images from figure 2. Pixel values have been rearranged from $[-25,25]$ to $[0,250]$ for better visibility. We observe that IHS, Brovey, Wavelet-based and $\mathrm{P}+\mathrm{XS}$ techniques lead to the loss of spectral information. Although a slight color distortion remains, the proposed algorithm is able to better preserve the chromacity of the original image.

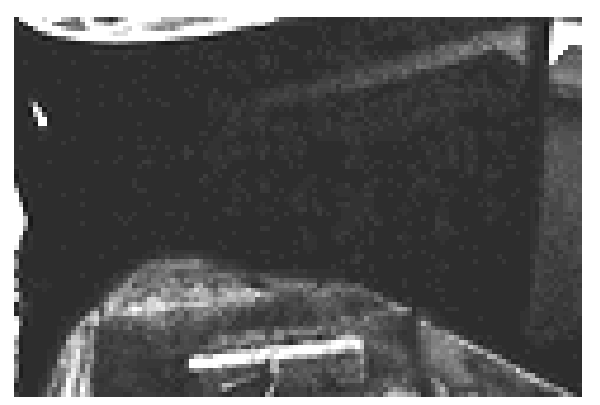

Original

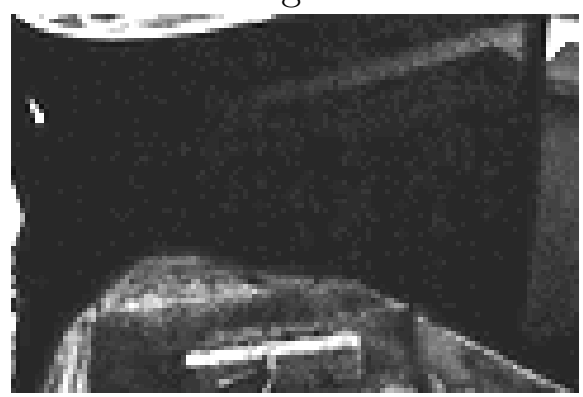

Wavelets

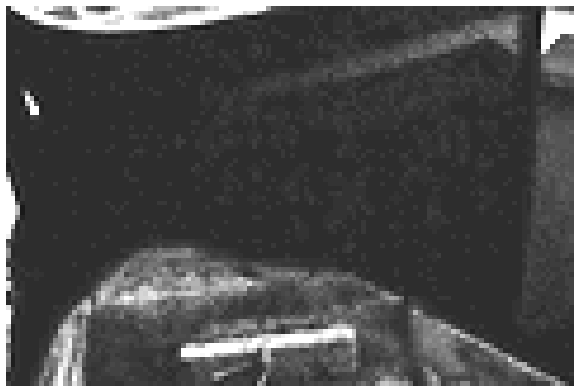

IHS

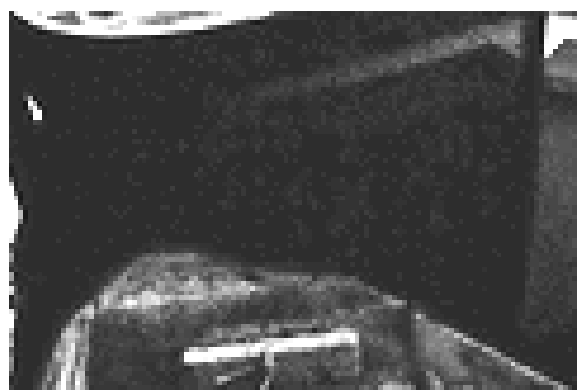

$\mathrm{P}+\mathrm{XS}$

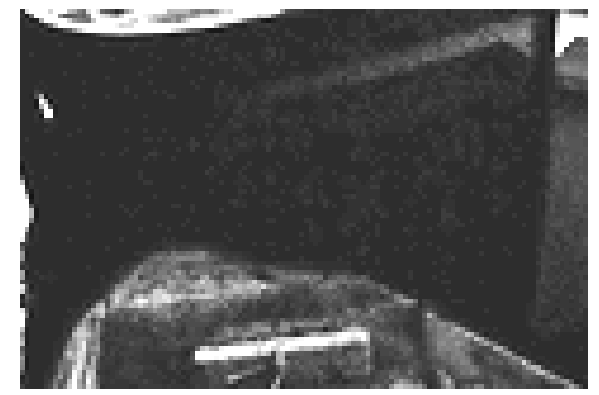

Brovey

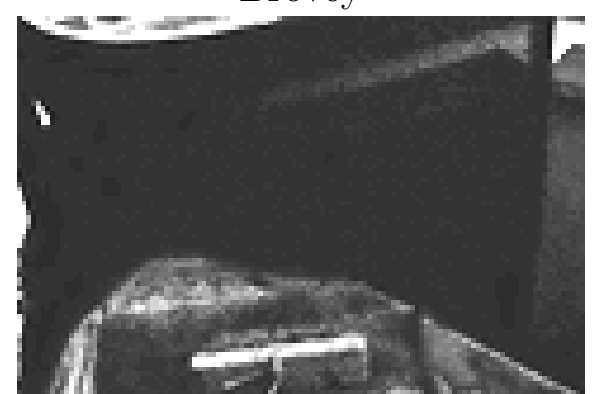

Duran et al.

Figure 4: Original panchromatic image and the grayscale versions of the pansharpened images provided by each technique, with sampling factor $s=4$. Pixel values have been rearranged to [0,50] for better visibility. All results compare favorably to the original panchromatic, so that edges, textures and other geometric details are highly preserved. Note that the proposed algorithm also performs denoising since it reduces the inherent noise that appears, for instance, at the bow hull of the boat. 


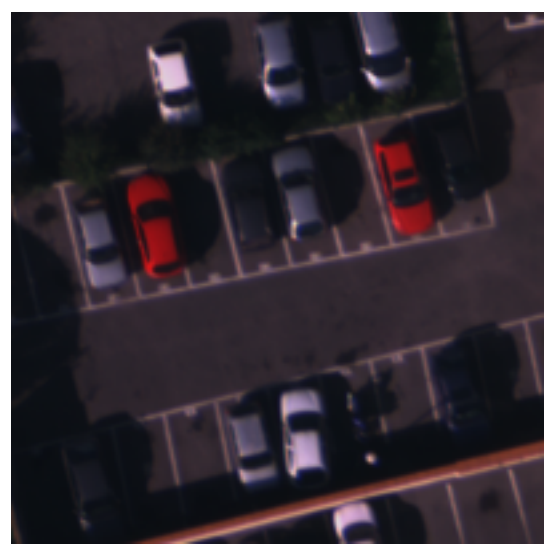

Original

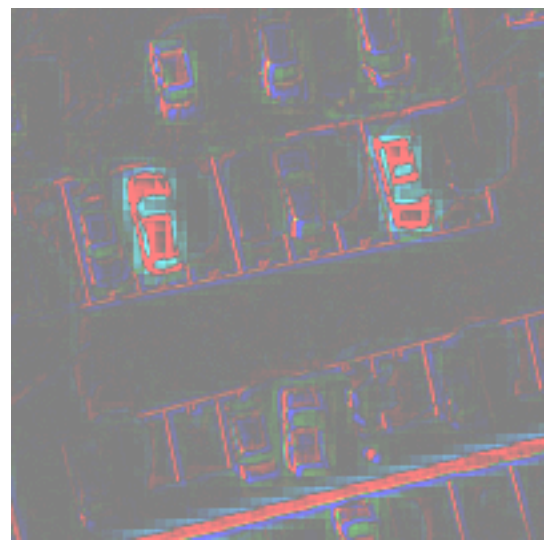

Wavelets

$\mathrm{RMSE}=4.51$

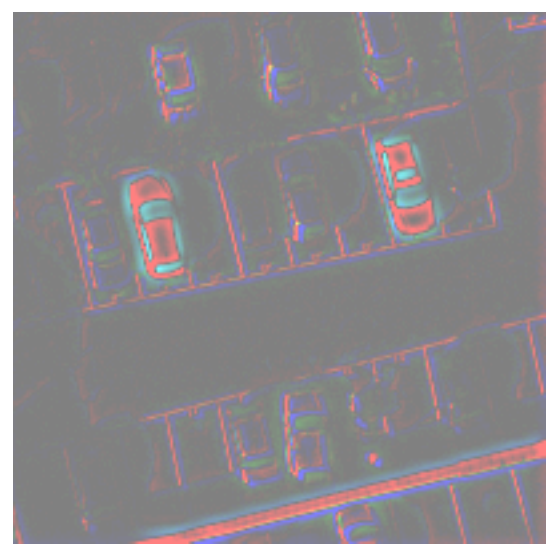

IHS

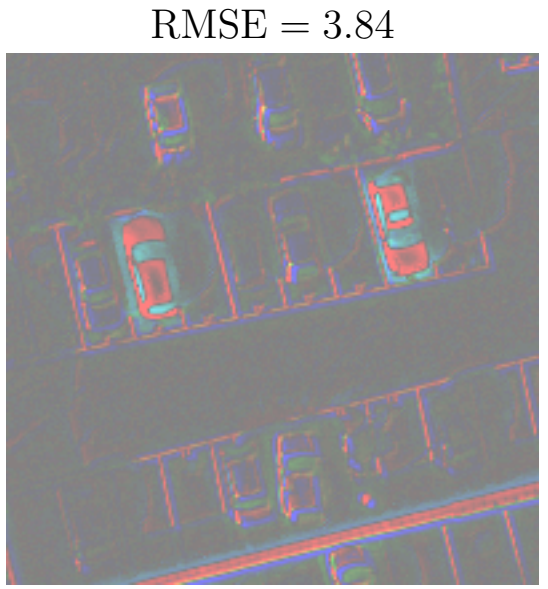

$\mathrm{P}+\mathrm{XS}$

$\mathrm{RMSE}=3.72$

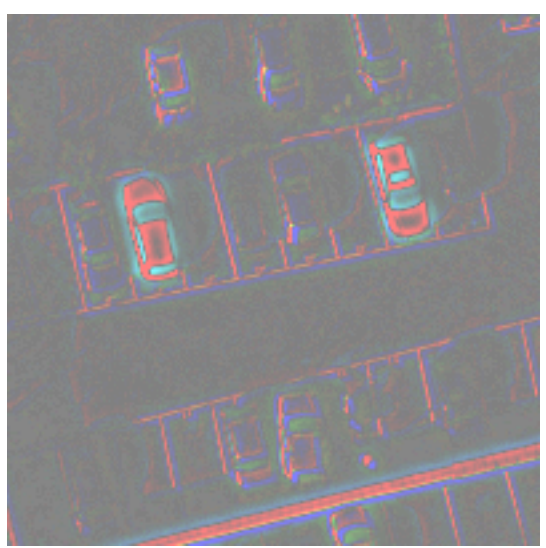

Brovey

$\mathrm{RMSE}=3.90$

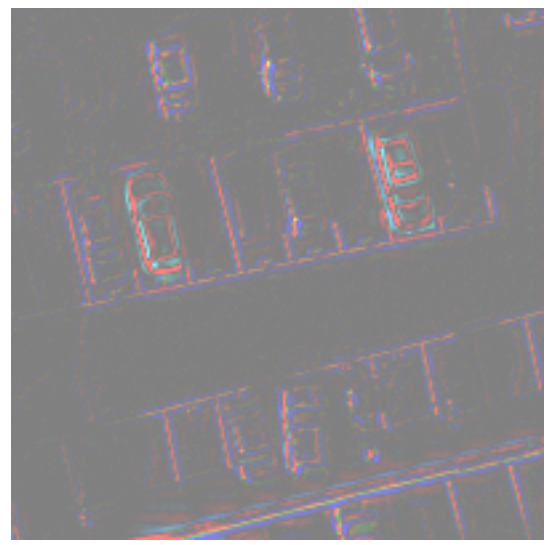

Duran et al.

$\mathrm{RMSE}=3.03$

Figure 5: Experiment with an RGB aerial image using $s=4$ as sampling factor. We exhibit the difference images between the reference one and the pansharpened results provided by each method under comparison. Pixel values have been rearranged from $[-25,25]$ to $[0,255]$ for better visibility. Furthermore, the RMSE values between the original image and the pansharpened ones are displayed. To a greater or lesser extent, we observe that IHS, Brovey, Wavelet-based and P+XS techniques lead to the loss of spectral information. For instance, the color saturation of reddish objects as cars have been significantly reduced on the results provided by these methods. On the contrary, the model proposed by Duran et al. [3] is able to better preserve the chromacity and, thus, the difference image contains less amount of information. 


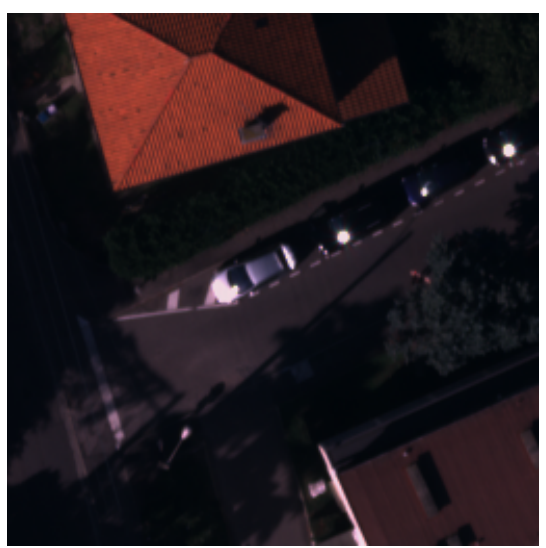

Original color image

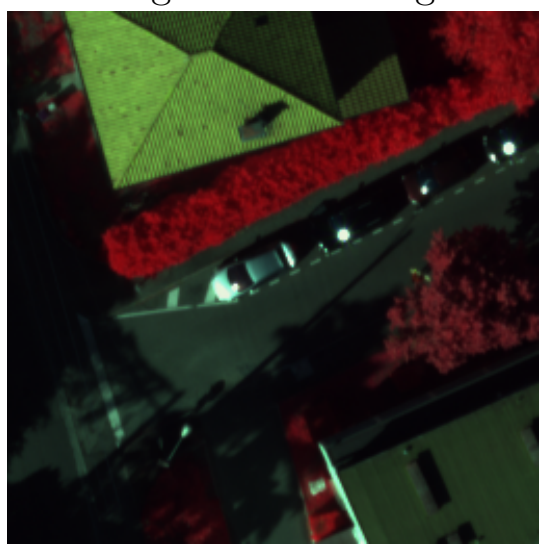

Original false color image

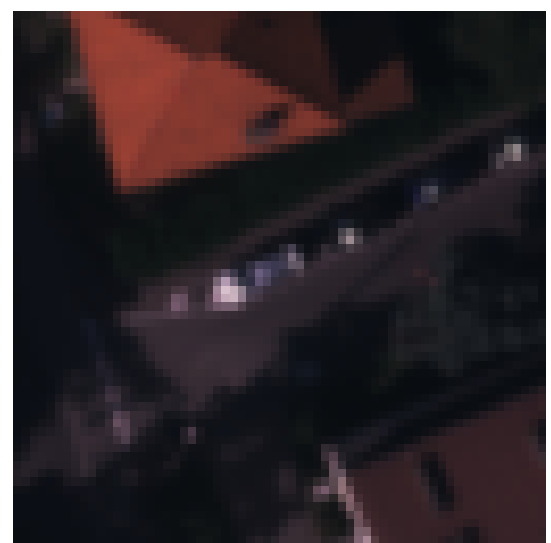

Low-resolution color image

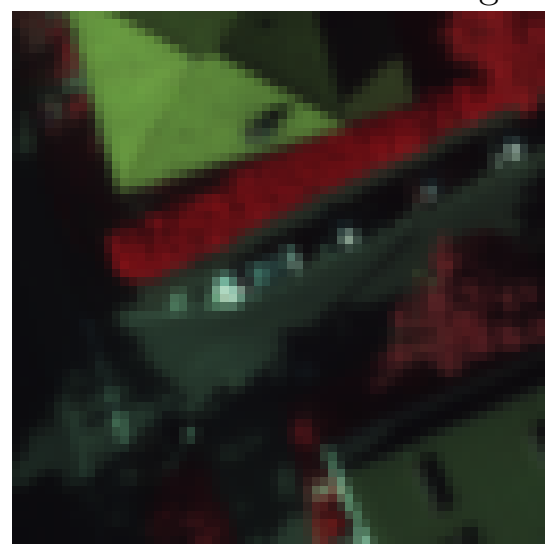

Low-resolution false color image

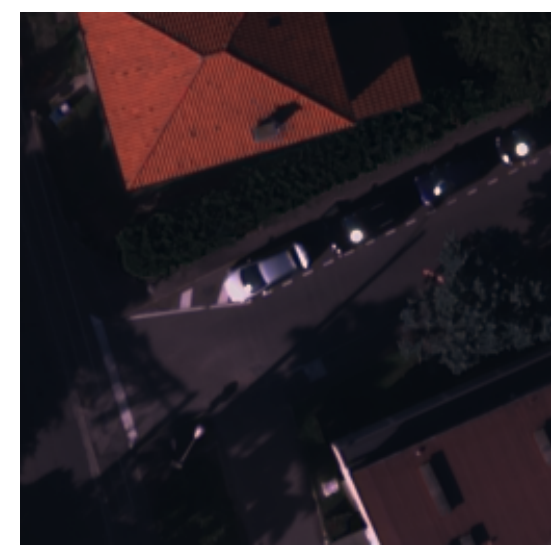

Pansharpened color image

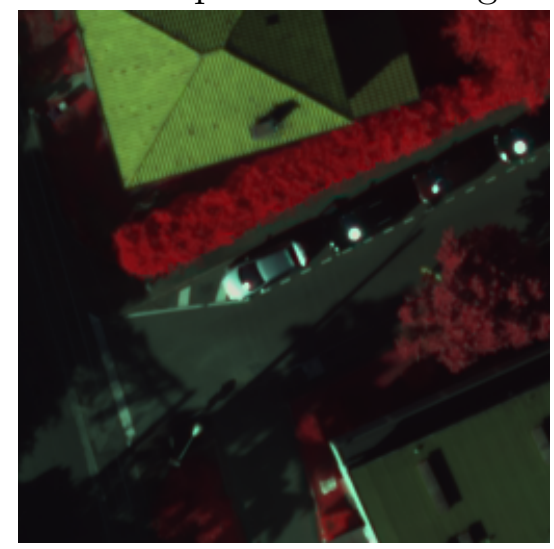

Pansharpened false color image

Figure 6: Test of algorithm 1 on a four band aerial image using $s=4$ as sampling factor. The first row displays the original, the low-resolution and the pansharpened color images involving red, green and blue channels. On the other hand, the second row exhibits the original, the low-resolution and the pansharpened false color images involving near-infrared, red and green spectral bands (in this order) in place of the usual RGB. The size of low-resolution images is $64 \times 64$ pixels, whereas the size of original and pansharpened images is $256 \times 256$ pixels. 


\section{Image Credits}

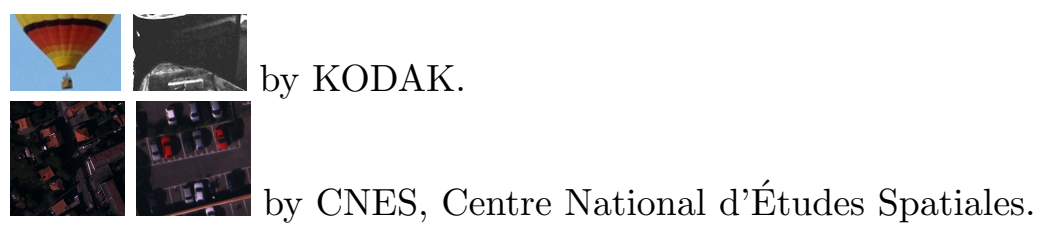

\section{References}

[1] B. Aiazzi, L. Alparone, S. Baronti, And A. Garzelli, Context-driven fusion of high spatial and spectral resolution images based on oversampled multiresolution analysis, IEEE Transactions on Geoscience and Remote Sensing, 40 (2002), pp. 2300-2317. http://dx.doi.org/10. 1109/TGRS. 2002.803623.

[2] C. Ballester, V. Caselles, L. Igual, and J. Verdera, A variational model for $P+X S$ image fusion, International Journal of Computer Vision, 69 (2006), pp. 43-58. http://dx. doi . org/10.1007/s11263-006-6852-x.

[3] A. Buades, B. Coll, J. Duran, and C. Sbert, A nonlocal variational model for pansharpening image fusion, (2014). Manuscript in press in SIAM Journal on Imaging Sciences.

[4] A. Buades, B. Coll, And J.-M. Morel, A review of image denoising algorithms, with a new one, SIAM Multiscale Modeling and Simulation, 4 (2005), pp. 490-530. http://dx.doi. org/10.1137/040616024.

[5] J. W. Carper, T. M. Lillesand, and R. W. Kiefer, The use of intensity-hue-saturation transformations for merging SPOT panchromatic and multispectral image data, Journal of Photogrammetric Engineering and Remote Sensing, 56 (1990), pp. 459-467.

[6] P. S. Chavez, S. C. Sides, And J. A. Anderson, Comparison of three different methods to merge multiresolution and multispectral data - landsat TM and SPOT panchromatic, Journal of Photogrammetric Engineering and Remote Sensing, 57 (1991), pp. 295-303.

[7] G. Cliche And F. Bonn, Integration of SPOT panchromatic channel into multispectral mode for image sharpness enhancement, Journal of Photogrammetric Engineering and Remote Sensing, 51 (1985), pp. 311-316.

[8] Q. Du, N. H. Younan, R. King, And V. P. Shah, On the performance evaluation of pansharpening techniques, IEEE Geoscience and Remote Sensing Letters, 4 (2007), pp. 518-522. http://dx.doi.org/10.1109/LGRS .2007.896328.

[9] M. Ehlers, Multisensor image fusion techniques in remote sensing, International Society for Photogrammetry and Remote Sensing, 46 (1991), pp. 19-30. http://dx.doi.org/10.1016/ 0924-2716 (91) 90003-E.

[10] B. Garguet-Duport, J. Girel, J. M. Chassery, and G. Pautou, The use of multiresolution analysis and wavelet transform for merging SPOT panchromatic and multispectral image data, Journal of Photogrammetric Engineering and Remote Sensing, 62 (1996), pp. 1057-1066.

[11] G. Gilboa And S. J. Osher, Nonlocal image regularization and supervised segmentation, SIAM Multiscale Modeling and Simulation, 6 (2007), pp. 595-630. http://dx.doi.org/10. $1137 / 060669358$. 
[12] R. Haydn, G. W. Dalke, J. Henkel, And J. E. Bare, Applications of the IHS color transform to the processing of multisensor data and image enhancement, in Proceedings of the International Symposium on Remote Sensing of Arid and Semi-Arid Lands, Cairo, Egypt, 1982, pp. 599-616.

[13] S. Kindermann, S. Osher, And P. W. Jones, Deblurring and denoising of images by nonlocal functionals, SIAM Multiscale Modeling and Simulation, 4 (2005), pp. 1091-1115. http: //dx.doi.org/10.1137/050622249.

[14] M. Mahmoudi And G. Sapiro, Fast image and video denoising via nonlocal means of similar neighborhoods, IEEE Signal Processing Letters, 12 (2005), pp. 839-842. http://dx.doi.org/ 10.1109/LSP. 2005.859509.

[15] M. Möller, T. Wittman, A. L. Bertozzi, and M. Burger, A variational approach for sharpening high dimensional images, SIAM Journal on Imaging Sciences, 5 (2012), pp. 150-178. http://dx.doi.org/10.1137/100810356.

[16] J. Nunez, X. Otazu, O. Fors, A. Prades, V. PalÀ, and R. Arbiol, Multiresolutionbased image fusion with additive wavelet decomposition, IEEE Transactions on Geoscience and Remote Sensing, 37 (1999), pp. 1204-1211. http://dx.doi.org/10.1109/36.763274.

[17] C. Pohl and J. L. Genderen, Multisensor image fusion in remote sensing: concepts, methods and applications, International Journal of Remote Sensing, 19 (1998), pp. 823-854. http: //dx.doi.org/10.1080/014311698215748.

[18] L. I. Rudin, S. J. Osher, And E. FAtemi, Nonlinear total variation based noise removal algorithms, Physica D: Nonlinear Phenomena, (1992), pp. 259-268. http://dx.doi.org/10. 1016/0167-2789(92) 90242-F.

[19] V. K. Shettigara, A generalized component substitution technique for spatial enhancement of multispectral images using a higher resolution data set, Journal of Photogrammetric Engineering and Remote Sensing, 58 (1992), pp. 561-567.

[20] C. Thomas, T. Ranchin, L. Wald, and J. Chanussot, Synthesis of multispectral images to high spatial resolution: A critical review of fusion methods based on remote sensing physics, IEEE Transactions on Geoscience and Remote Sensing, 46 (2008), pp. 1301-1312. http://dx. doi.org/10.1109/TGRS. 2007.912448.

[21] T.-M. Tu, P. S. Huand, C.-L. Hung, And C.-P. Chan, A fast intensity-hue-saturation fusion technique with spectral adjustment for IKONOS imagery, IEEE Geoscience and Remote Sensing Letters, 4 (2004), pp. 309-312. http://dx.doi.org/10.1109/LGRS.2004.834804.

[22] V. Vijayaraj, C. G. O. Hara, And N. H. Younan, Quality analysis of pansharpened images, in Proceedings of the IEEE International Geoscience and Remote Sensing Symposium (IGARSS04), Anchorage, Alaska, 2004, Proceedings of the, pp. 85-88. http://dx.doi.org/ 10.1109/IGARSS . 2004.1368951.

[23] S. Cox W. A. Hallada, Image sharpening for mixed spatial and spectral resolution satellite systems, in Proceedings of the 17th International Symposium on Remote Sensing of Environment, Ann Arbor, Michigan, 1983, Proceedings of the, pp. 1023-1032. 
[24] T. A. Wilson, S. K. Rogers, And M. Kabrisky, Perceptual-based image fusion for hyperspectral data, IEEE Transactions on Geoscience and Remote Sensing, 35 (1997), pp. 1007-1017. http://dx.doi.org/10.1109/36.602543.

[25] Zhang Y, A new merging method and its spectral and spatial effects, International Journal of Remote Sensing, 20 (1999), pp. 2003-2014. http://dx.doi.org/10.1080/014311699212317.

[26] D. A. Yocky, Image merging and data fusion by means of the discrete two-dimensional wavelet transform, Journal of the Optical Society of America A, 12 (1995), pp. 1834-1841. http: //dx.doi.org/10.1364/JOSAA.12.001834.

[27] J. Zhou, D. L. Civico, And J. A. Silander, A wavelet transform method to merge landsat TM and SPOT panchromatic data, International Journal on Remote Sensing, 19 (1998), pp. 743757. http://dx.doi.org/10.1080/014311698215973. 